\title{
Current Status and Implementation of Pyramid Truth Wavefront Sensor on NFIRAOS Simulation Bench at NRC-Herzberg
}

\author{
Etsuko Mieda ${ }^{a}$, Jean-Pierre Veran ${ }^{a}$, Olivier Lardiere ${ }^{a}$, Siqi Liu $^{b}$, Masen Lamb $^{c}$, Paolo Turri $^{c}$, \\ David Andersen ${ }^{a}$, Glen Herriot ${ }^{a}$ \\ ${ }^{a}$ National Research Council Herzberg Astronomy and Astrophysics, 5071 W Saanich Rd., \\ Victoria, BC, Canada; \\ ${ }^{b}$ University of Toronto, 50 St. George St., Toronto, ON, Canada; \\ ${ }^{c}$ University of Victoria, 3800 Finnerty Rd., Victoria, BC, Canada
}

\begin{abstract}
We describe the components and functions and report the recent upgrades/modifications of the HeNOS (Herzberg NFIRAOS Optical Simulator) bench, a NFIRAOS (Narrow Field InfraRed Adaptive Optics System) simulator bench at NRC-Herzberg. The recent upgrade includes the new natural guide star simulator, the new science camera, and the implementation of a truth wavefront sensor using a pyramid. The truth wavefront sensing simulation involves the reproduction of Shack-Hartmann wavefront sensor elongation due to the sodium layer profile, which we simulate with a set of defocus on the ground layer deformable mirror. This is the first truth wavefront sensor made with a pyramid in use today.
\end{abstract}

Keywords: Pyramid wavefront sensor, Thirty Meter Telescope

\section{INTRODUCTION}

NFIRAOS (Narrow Field InfraRed Adaptive Optics System) will be the first adaptive optics (AO) system to be deployed on TMT (Thirty Meter Telescope). NFIRAOS is a multi-conjugate AO system equipped with six laser guide stars (LGSs) and two deformable mirrors (DMs). Because the scale of NFIRAOS is unprecedented to test on pre-existing facilities, we build HeNOS (Herzberg NFIRAOS Optical Simulator) bench to simulate the performance and develop calibration procedures, such as non-common path aberration (NCPA) corrections. We also test real-time point-spread-function reconstruction (PSFR) on the MCAO bench, ${ }^{1}$ which is a crucial functionality enabling quantitative astronomy with $\mathrm{AO}$ but has proven to be a challenging problem.

A brief description of the bench parameters can be found in $\S 2$, including the new design of a natural guide star source simulator $(\S 2.1)$ and the upgrade of the science camera $(\S 2.2)$. The new implementation of a truth wavefront sensor made with a pyramid can be found in $\S 3$. In $\S 4$, we summarize the bench and discuss the future plan.

\section{BENCH DESCRIPTION}

In this section, we briefly describe the components of the HeNOS bench and report the most updated status since the last updates, ${ }^{2,3}$ including the NGS light source modification and the science camera upgrade. The HeNOS bench consists of four LGSs, a grid of natural guide stars (NGSs), two DMs, three phase screens (PSs), one Shack-Hartmann wavefront sensor (SHWFS), one science camera (SC), and a truth WFS made with a pyramid (PTWFS). Figure 1 shows the optical paths, and Table 1 describes components and their abbreviations. The details of the bench design, parameters, and calibration procedures can be found in the references. ${ }^{2-6}$ To simplify the bench and calibrate it better, there are a couple of incomplete implementation in the bench. Currently a flat mirror is placed at the position of DM1, and only DM0 is in the path. Also we are working on a new NGS simulator (see $\S 2.1$ for detail), and thus the SC is placed at the LGS focus instead of NGS focus.

Send correspondence to Etsuko Mieda. Email: Etsuko.Mieda@nrc-cnrc.gc.ca 


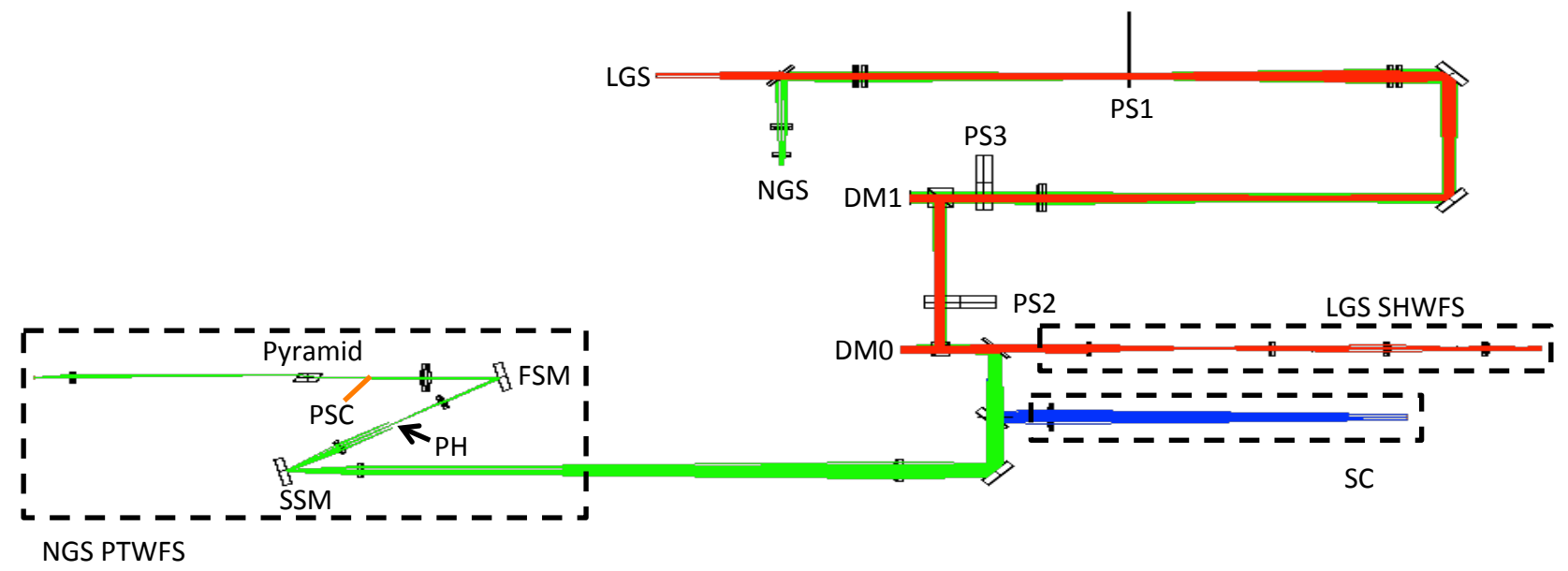

Figure 1 The optical paths of the HeNOS bench. The descriptions of abbreviations are listed in Table 1. The bench consists of 2 by 2 configuration LGSs, a grid of NGSs, two DMs conjugated to 0 and $12 \mathrm{~km}$, three PSs conjugated to 0.6, 5.2, and $16.3 \mathrm{~km}$, one SHWFS (red) simultaneously measuring four LGSs, one SC (blue), and TWFS made with a double pyramid (green). For PWFS performance evaluation, one more science camera focused on NGS, called PSC, is also added (orange).

Table 1. Bench Components

\begin{tabular}{ccl}
\hline \hline Abbrv & Name & \multicolumn{1}{c}{ Description } \\
\hline LGS & Laser Guide Star & 2 by 2 configuration lasers whose separation defines 4.5 arcsec on the sky. \\
NGS & Natural Guide Star & Creates a grid of NGSs, previously by microlens array, now by pinhole mask. \\
DM0 & Deformable Mirror 0 & ALPAO DM with 97 actuators at ground (0 km). \\
DM1 & Deformable Mirror 1 & ALPAO DM with 277 actuators at high altitude $(12 \mathrm{~km})$. \\
PS1 & Phase Screen 1 & UCSC (paint spraying) PS at ground layer $(0.6 \mathrm{~km})$. \\
PS2 & Phase Screen 2 & Lexitex (index matching) PS at middle layer $(5.2 \mathrm{~km})$. \\
PS3 & Phase Screen 3 & Lexitex (index matching) PS at high layer $(16.3 \mathrm{~km})$. \\
FSM & Fast Steering Mirror & Newport FSM-300. \\
PH & Pinhole & $500 \mu$ m pinhole at NGS focus to block all but one NGS. \\
SSM & Star Selection Mirror & Zaber motorized gimbal mount and mirror. \\
SHWFS & Shack-Hartmann WFS & 30 by 30 subapeture SHWFS with Pointgrey Grasshopper $(2448 x 2048,3.45 \mathrm{~m}$ pixel). \\
SC & Science Camera & Andor sCMOS Zyla (2048x2048, 6.5m pixel) currently at LGS focus. \\
PTWFS & Pyramid Truth WFS & 76 pixel diameter pupil PWFS with Pointgrey Flea $(638 \times 488,5.6 \mu \mathrm{m}$ pixel). \\
PSC & Pyramid Science Camera Pointgrey Grasshopper (2448 x 2048, 3.45 $\mu$ m pixel) at NGS focus. \\
\hline
\end{tabular}

\subsection{NGS Light Source Simulator}

To evaluate the AO performance over the whole science field, we created a grid of NGSs using a microlens array (MA). However, MA created a grid intensity pattern on a pupil (left on Figure 2). This grid pattern is probably created by the concentrated light from the flat part of the glass between microlenses on top of a pupil (right on Figure 2).

We are currently working on a new design without MA. The new design consists of a powerful LED, collimating lens, diffuser, and a pinhole mask in a lens tube. See Figure 3 on the left for the schematic description of this new design and on the right for the test setup.

To produce a PSF, the size of the pinhole has to be smaller than the diffraction limit of the bench, which is $\sim 10 \mu \mathrm{m}$. Unlike MA, this tiny pinhole blocks most of photons and makes it difficult to have good signal at the 


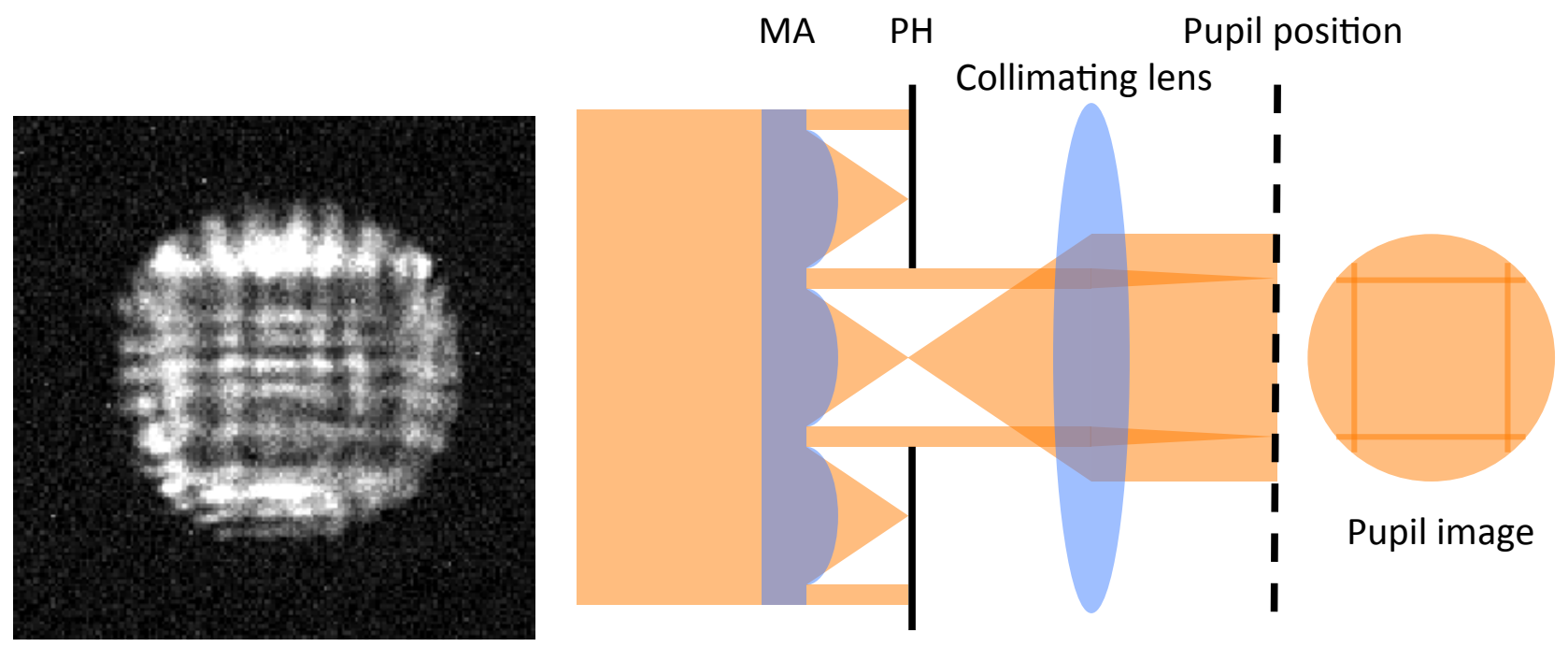

Figure 2 An image of a grid pattern pupil from the old NGS light source with MA (left). The grid pattern is probably the concentrated light from the flat part of the glass between microlenses on top of a pupil (right).

detector. Unfortunately, the HeNOS bench uses many beamsplitters, including two cube beamsplitters in front of DMs, most photons are wasted, and the pinhole mask reduce them even lower. We are adjusting the position of collimating lens and pinhole mask to maximizing the number of photons at the pinhole mask, and changing the beamsplitters with different reflection-transmission ratios to give more weight on NGS light.

We are also looking into a design where the pinhole mask can be replaced with a single pinhole of a large radius. By using a bigger pinhole, we can experiment a wavefront sensing on an extended objects using PWFS. See this reference ${ }^{7}$ for extended object simulations and science applications.

\subsection{Science Camera Upgrade}

We originally used Pointgrey Grasshopper (2448 x 2048, $3.45 \mu \mathrm{m}$ pixel), the same camera as SHWFS. However, we realized that the Grasshopper has two readout channels which create background offset between the two sides
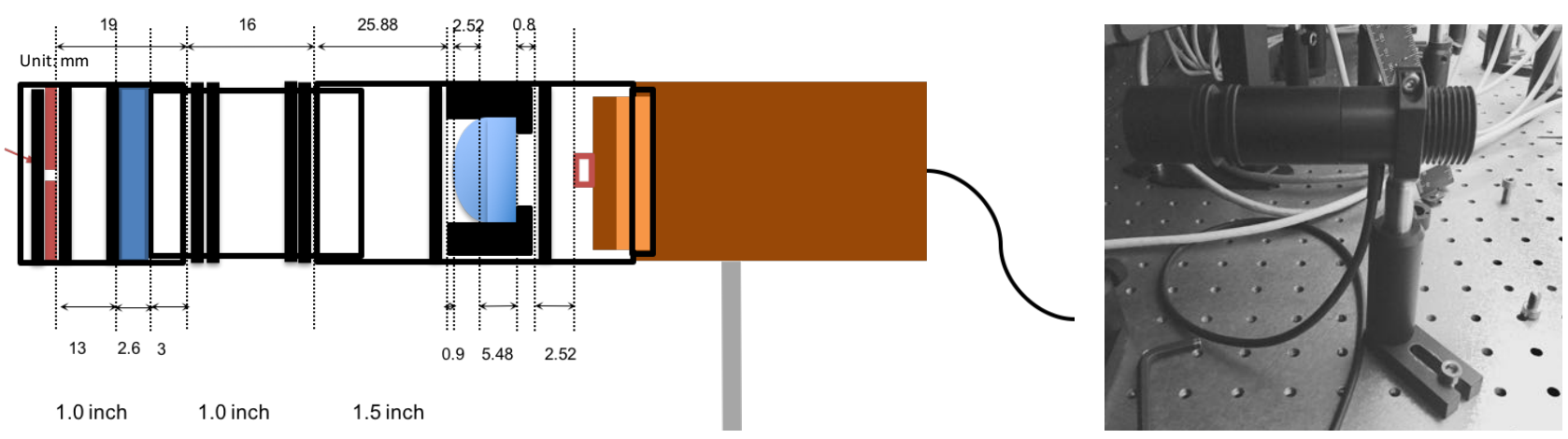

Figure 3 New NGS light source design schematic (left) and its test setup on the bench (right). The new design uses a pinhole mask instead of MA. The difficulty with this design is that unlike MA, pinholes black most of photons, and photons reaching the detector is very limited. 

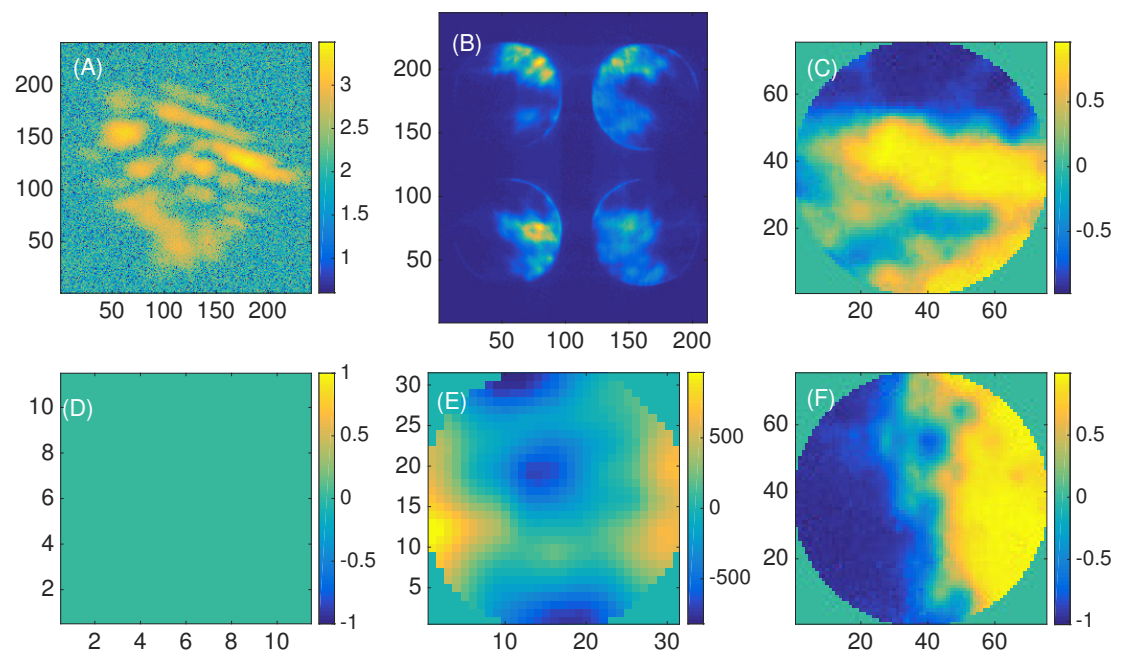

Figure 4 Zero command is applied to DM0 and measured with PTWFS. (A) PSF on PSC, (B) raw pupil image, (C) $\mathrm{x}$ - and (F) y-slope signal, (D) DM0 shape, and (E) reconstructed wavefront are shown.

of the detector. This is a problem when we do the PSF reconstruction experiment. We thus replaced the camera with Andor sCMOS Zyla (2048 x 2048, $6.5 \mu \mathrm{m}$ pixel). Andor sCMOS has almost the same number of pixels but with bigger pixel, so we realigned the lenses in front of it to have a right magnification.

Due to the NGS light source problem (§2.1), currently we do not have a proper NGS light source on the bench. However, while we work on new solutions, we would like to continue running experiments on the bench. To temporary solve the situation, we locate SC at the focus of LGSs, and use LGSs as science targets. At this location, the plate scale for LGSs is 5.6 milli-arcsec (mas).

\section{PYRAMID TRUTH WAVEFRONT SENSOR}

The one major update to the HeNOS bench is the implementation of a truth WFS made with PWFS (green path in Figure 1). The description of TWFS and the HeNOS TWFS design are reported in the reference, ${ }^{3}$ and the final design is shown in Figure 1 in green. In short, the grid of NGSs hitting Star Selection Mirror (SSM) at
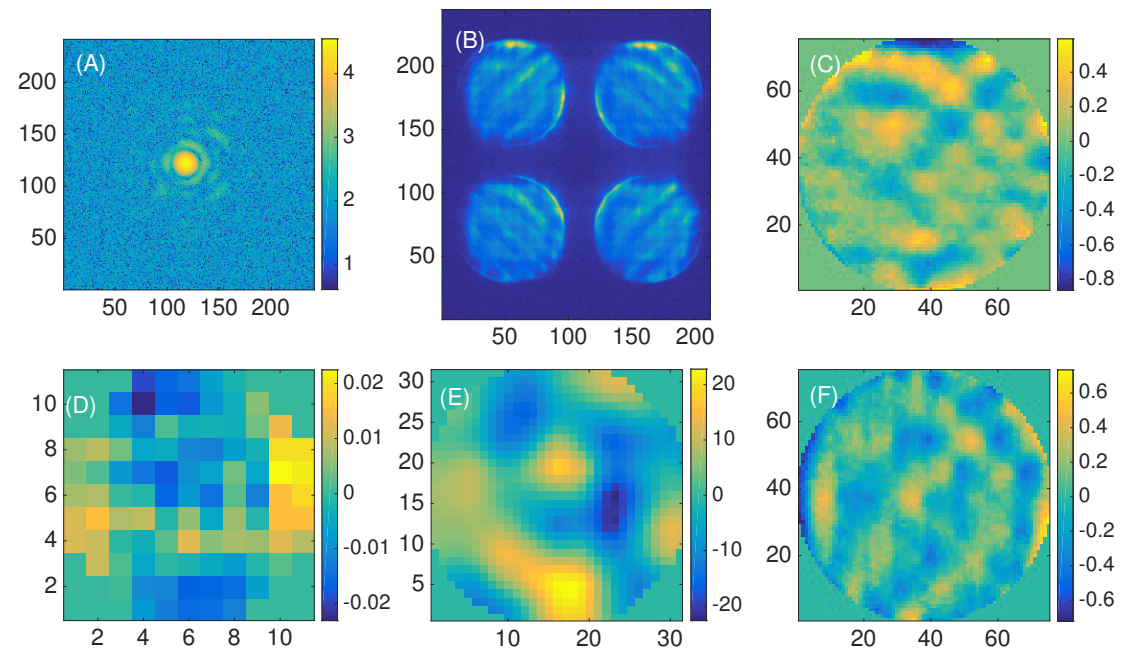

Figure 5 Starting from zero command (Figure 4), the loop is cosed after 50 iterations with loop gain $=0.2$, and diffraction rings are seen in the PSF (panel A). See Figure 4 caption for the descriptions of each panel. 


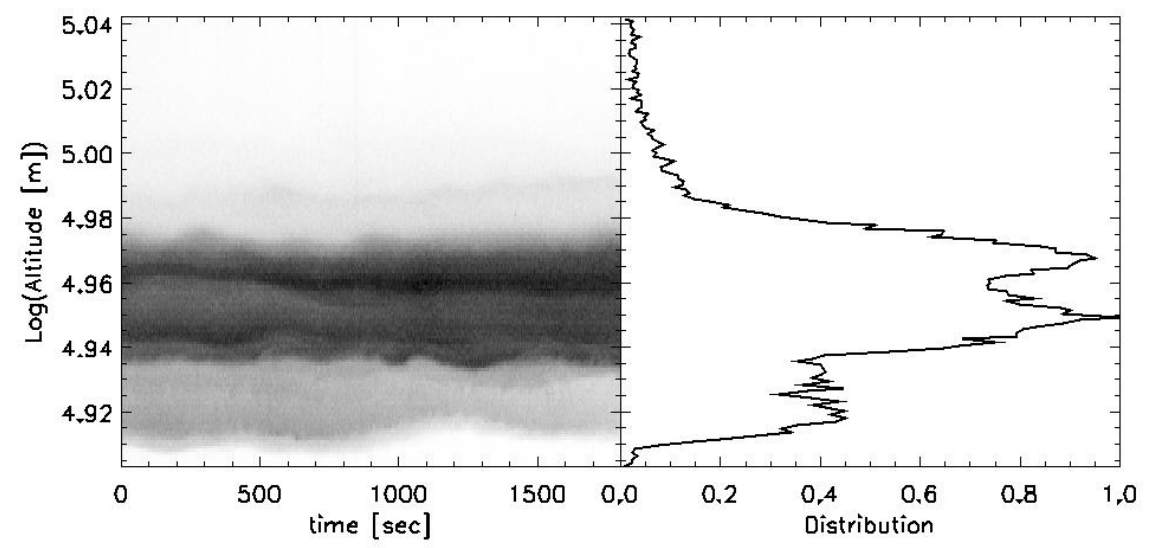

Figure 6 Left: the empirically obtaned Na profile for 1800 seconds. Darker color for higher density. Right: 1D plot of $\mathrm{Na}$ profile at $t=0$ second.

pupil is sent to Pinhole (PH) where only one NGS goes through. A beam from the single NGS is then modulated by Fast Steering Mirror (FSM) at pupil, and the focused beam makes a circle around the vertex of the pyramid. The bench now can be closed with this PWFS. As an example, the PSF, raw detector image, $\mathrm{x}$ - and y-slope signal, DM0 shape, and recostructed wavefront before (Figure 4, zero commands on DM0) and after (Figure 5) the loop is closed are shown. To evaluate the PSF at the tip of the pyramid, we have inserted one more beamsplitter between the focusing lens and the pyramid and added a camera (pyramid science camera, PSC, orange path in Figure 1). The initial PSF is big and fuzzy, and after closing the loop, the diffraction rings are visible.

\subsection{SHWFS Elongation}

When Sodium (Na) laser is used for LGS, the spots on SHWFS are radially elongated due to the finite thickness of the Na layer. When the Na layer profile (i. e., height, thickness, and density distribution) changes, the photon distribution over the elongated spots also changes. This intensity changes along the radially elongated spot introduce an additional centroid shift when the basic centroding method is applied. See for example this reference $^{3}$ for more detail about how SHWFS elongation and imperfection in centroiding.

To simulate the SHWFS elongation created by the sodium layer profile and thickness, we apply a set of defocus commands to DM0 while change the LGS intensity according to the empirically obtained Na profile. An
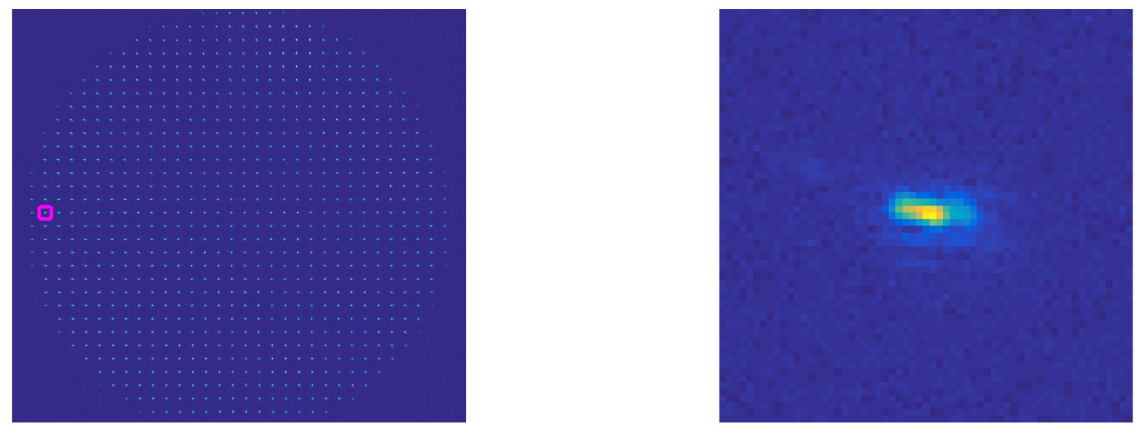

Figure 7 Simulated elongated SHWFS spots when Na profile at $t=0$ (right on Figure 6 ) is applied. Full frame SHWFS camera image is shown on the left, and one zoomed spot in a magenta box is shown on the right. 

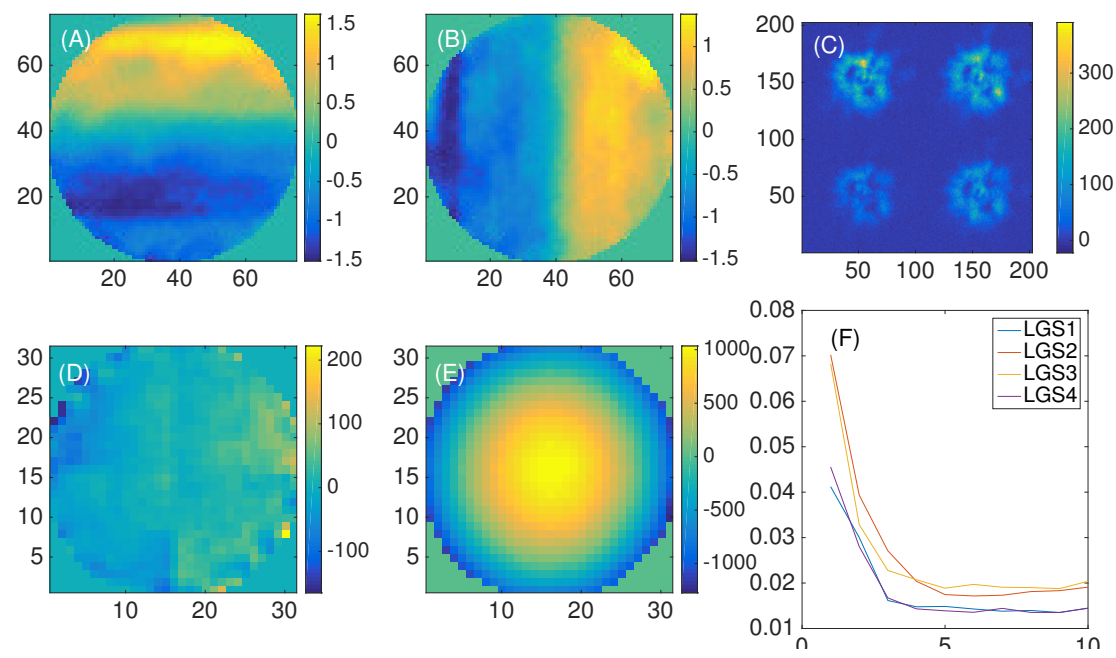

Figure 8 After closing loop on elongated SHWFS spots with Na profile shown in the right panel of Figure 6 without TWFS feedback. (A) PWFS slope signal in x, (B) PWFS slope signal in y, (C) four LGSs on the science camera, (D) reconstructed phase using elongated SHWFS, (E) reconstructed phase using normal SHWFS, (F) evolution of four LGS Strehl ratios while closing loop.

example of empirical Na profile for 1800 seconds and reproduced SHWFS elongation at $t=0$ second are shown in Figure 6 and 7, respectively.

\subsection{Close Loop with Truth Wavefront Sensor}

On the HeNOS bench, we close the loop using the elongated SHWFS spots and PWFS as TWFS. Each iteration, we apply a set of defocus command to simulate elongated SHWFS image and send it to a centroinding code, which we currently use a basic center of gravity method. Without any correction, the resultant spot positions are biased according to the Na profile, and the new DM commands obtained from this biased positions include radial aberrations. The new DM command is applied to DM0, and PWFS operates wavefront sensing on NGS. In TMT+NFIRAOS case, TWFS does not measure the defocus term and only measures higher order radial
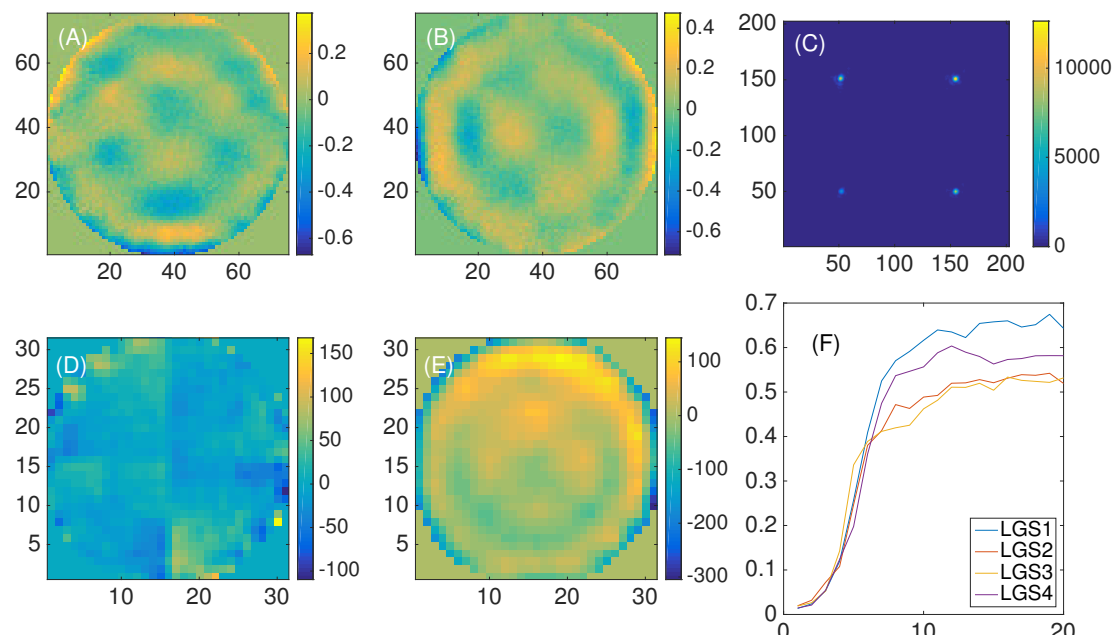

Figure 9 After closing loop on elongated SHWFS spots with Na profile shown in the right panel of Figure 6 with TWFS feedback. See Figure 8 caption for descriptions of panels. 
modes, but on the HeNOS bench, we measure the defocus term as well because we do not have a separate focus wavefront sensor.

Figure 8 and 9 shows a result of closing loop with elongated SHWFS. Starting with the best DM flat from the previous day, the loop is closed using the elongated SHWFS spots. For this experiment, we apply the same $\mathrm{Na}$ profile shown in Figure 6 on the right for all iteration (no Na profile evolution). We use a higher loop gain of 0.5 for this test to speed up the simulation. Before the TWFS feedback is applied (Figure 8), because the loop is closed to the Na profile, the reconstructed phase by elongated SHWFS (panel D) sees flat phase while the actual phase (panel E) include a big defocus and radial aberrations. The defocus is also seen on PWFS (panel A and B). Panel F shows four LGS Strehl ratios become low, and well before 10th iteration, the value plateau. The final PSFs on panel $\mathrm{C}$ are big and fuzzy.

After the loop is closed on the elongated SHWFS with Na profile, we then close loop again but now with TWFS feedback (Figure 9). The TWFS feedback is applied as a reference slope to the elongated SHWFS. After 20 iterations, the four LGS Strehl ratios (panel F) are improved (> 50\%), and all three WFSs, PWFS (panel A and B), elongated SHWFS (panel D), and normal SHWFS (panel E) see small aberrations.

\section{SUMMARY AND FUTURE PLAN}

We have reported the recent upgrades and modifications to the HeNOS bench, including the new NGS light source simulator $(\S 2.1)$ and the new science camera $(\S 2.2)$. The biggest upgrade is the addition of the TWFS made with a PWFS (§3). The truth wavefront sensing includes simulating SHWFS spot elongation due to the Na thickness and density profile, and PWFS feedback as a reference slope to the SHWFS. The SHWFS elongation is simulated by modulating the ground layer DM and LGS laser intensities according to the empirically measured Na profile. Our TWFS is the first one in use made with a PWFS.

There are several hardware and software additions to be completed soon. For hardware additions, we have started to work on a new NGS source simulator design using a pinhole mask (§2.1). We will make more measurement with the prototype (Figure 3) and finalize the design. Once the new NGS light source is built, then we can move the new science camera back to the NGS focus position. Currently, we use a flat mirror instead of a DM at the high altitude DM position to simplify the bench for calibration purposes. Most calibrations are done, and we expect to implement the DM soon.

For the software upgrade, we are working on including a matched filter centroiding method. Also right now, we close the loop only with one LGS, thus it is a single conjugate AO loop. We are collaborating with LAM on implementing a laser tomography AO functionality to HeNOS, which can be achieved without having the second DM. Once we add the second DM, we can also include multi-conjugate AO.

\section{ACKNOWLEDGMENTS}

We would like to thank Tim Hardy and Jonathan Stocks at NRC-Herzberg for their help developing both hardware and software.

\section{REFERENCES}

1. L. Gilles, L. Wang, E. Mieda, V. J.-P., M. Schock, B. Ellerbroek, C. Boyer, and G. Trancho, "Point Spread Function Reconstruction Simulations \& Tests on the HeNOS Bench," in Adaptive Optics for Extremely Large Telescopes 5, July 2017.

2. M. Rosensteiner and J.-P. Véran, "Testing the analytical model of the pyramid wavefront sensor with highorder aberrations on the optical bench," in Adaptive Optics Systems IV, 9148, p. 914853, July 2014.

3. E. Mieda, M. Rosensteiner, M. van Kooten, J.-P. Veran, O. Lardiere, and G. Herriot, "Testing the pyramid truth wavefront sensor for NFIRAOS in the lab," in Adaptive Optics Systems V, 9909, p. 99091J, July 2016.

4. J.-P. Véran, E. McWeigh, D. Andersen, C. Correia, G. Herriot, and J. Pazder, "The HIA MCAO laboratory bench," in Adaptive Optics Systems III, 8447, p. 844750, July 2012.

5. P. Turri, D. R. Andersen, J.-P. Véran, P. Spanò, M. Rosensteiner, and E. A. McVeigh, "An MCAO test bench for NFIRAOS," in Adaptive Optics Systems IV, 9148, p. 91485Y, Aug. 2014. 
6. M. Rosensteiner, P. Turri, D. Andersen, P. Spano, and G. Herriot, "Laboratory tests on HeNOS, the MCAO test bench for NFIRAOS," in Adaptive Optics for Extremely Large Telescopes 4, Oct. 2015.

7. E. Mieda, J. Fung, and V. J.-P., "Simulating the Performance of Pyramid Wavefront Sensors on Extended Objects and Broadband Sensing," in Adaptive Optics for Extremely Large Telescopes 5, July 2017. 